\title{
A MATHEURISTIC-BASED APPROACH FOR THE MULTI-DEPOT HOME HEALTH CARE ASSIGNMENT, ROUTING AND SCHEDULING PROBLEM
}

\author{
Jérémy Decerle ${ }^{1, *}$, Olivier Grunder ${ }^{2}$, Amir Hajuam El Hassani ${ }^{2}$ And \\ OUSSAMA BARAKAT ${ }^{3}$
}

\begin{abstract}
Home health care structures provide care for the elderly, people with disabilities as well as patients with chronic conditions. Since there has been an increase in demand, organizations providing home health care are eager to optimize their activities. In addition, the increase in patient numbers has led organizations to expand their geographical reach. As a result, home health care structures tend to be located in different offices to limit their travel time and, consequently, caregivers employed by these various structures must be assigned to one of the offices so they start and end their workday at their associated office. Unlike the existing literature where an upstream assignment of caregivers is performed to become a parameter of the model, the assignment of caregivers to offices is solved during the resolution of the problem in order to obtain the best possible combinations. Thus, we suggest a mixed-integer programming model of the multi-depot home health care assignment, routing, and scheduling problem without prior assignment of caregivers to the home health care offices. In addition, we propose an original matheuristic-based approach with different assignment strategies to assign visits and caregivers to the home health care offices in order to solve the problem. The experiments are conducted on a set of 56 heterogeneous instances of various sizes. Results are compared with best solutions obtained by a commercial solver, and with a lower bound obtained by Lagrangian relaxation. The results highlight the efficiency of the matheuristic-based approach since it provides a low deviation ratio with a faster computational time.
\end{abstract}

Mathematics Subject Classification. 90B06.

Received September 20, 2019. Accepted May 28, 2020.

\section{INTRODUCTION}

Home health care (HHC) services provide nursing and general hygiene care at patient homes for people in a situation of dependency. Different types of care are performed depending on the need of the patients. The caregivers at the home health care structures are often composed of nurses and auxiliary nurses, in order to provide the full range of care necessary. Nowadays, the planning of the caregivers is performed manually by an experienced nurse who has taken on the additional role of coordinator. This complex planning often requires

Keywords. Home health care, matheuristic, multi-depot, assignment, Lagrangian relaxation.

1 Institute for Transport Planning and Systems, ETH Zurich, Zurich 8093, Switzerland.

2 Nanomedicine Lab, Univ. Bourgogne Franche-Comté, UTBM, Belfort 90010, France.

3 Nanomedicine Lab, Univ. Bourgogne Franche-Comté, Besançon 25000, France.

*Corresponding author: jeremy.decerle@ivt.baug.ethz.ch 
extensive effort and time in order to obtain a valid schedule which respects all the constraints (availability of the patients, right qualification of the caregiver, ...). Since this task is performed daily, an important improvement is possible in favor of a more efficient schedule for caregivers.

However, the home health care problem remains difficult to solve as it falls into the category of NP-hard problems. Indeed, it is a variant of the vehicle routing problem, which has earlier been proven to be NP-hard [30].

The home health care problem was initially studied by Cheng and Rich [7] as a vehicle routing problem with time window and multiple depots.

Other characteristics have later been studied such as patient inconvenience, preferences, temporal dependencies between two visits, overtime cost of work, ... by Mankowska et al. [23] and Braekers et al. [5]. In many cases, the research is based on a real-life case study such as the decision support systems developed in Sweden by Eveborn et al. [16] and in the United States by Begur et al. [3].

A broader overview of the existing articles on the home health care problem can be obtained by the recent literature reviews proposed by Fikar and Hirsch [17] and Cissé et al. [8] which present a complete state of the art.

Recently, the synchronization of visits has appeared in many studies. Indeed, some care implies the simultaneous presence of two caregivers at the patient's home and there is therefore a need to synchronize the schedules of some of the caregivers during the day. In their study, Di Mascolo et al. [12] used a common technique to solve the synchronization constraint by creating a duplicate of these synchronized visits, then limiting the time that these two visits must be performed. In addition, the two duplicate visits have the same characteristics, except the qualification required by the caregivers to perform the care. This is particularly the case when the synchronized visit needs a nurse and an auxiliary nurse at the same time. Synchronization constraints have also been studied by Parragh and Doerner [25] who compare several ways of integrating a synchronization component into a metaheuristic algorithm for solving several routing and scheduling problems. In the context of vehicle routing problems, synchronization between vehicles' routes have also been studied by Liu et al. [22]. Since the addition of synchronization makes the problem more challenging than standard vehicle routing problem with time windows, they were especially interested in designing efficient neighborhood search-based methods for this kind of problem.

Due to the overall ageing of the population, the current strong demand will only increase as reported by Villeneuve and MacDonald [32] in Canada and Bertrand [4] in France. To that end, home health care structures are eager to optimize their activities, leading to an increased interest in the research on this topic. Consequently, many research articles have emerged from different countries such as Germany, Belgium and Denmark respectively studied by Nickel et al. [24], Duque et al. [14] and Rasmussen et al. [26]. As the demand is rising, the patients are increasingly spread out over the territory. Therefore, the home health care companies tend to be located in different offices in order to get closer to their patients.

However, one aspect of the home health care problem has received less attention so far. As related by Cissé et al. [8] in their literature review, the management as well as the organization of the caregivers is mostly left out by considering a single-district like [23]. It is actually the simplest alternative, in which the provider does not split caregivers into smaller clusters. However, some works have considered a multiple-district case where caregivers are assigned to an area and may only serve patients from this area. As a result, each district can be managed independently like the decision support system suggested by Duque et al. [14]. In their operational system for home care staff planning, Eveborn et al. [16] have imposed several restrictions including that each staff member be designated several working areas. Therefore, some patients can't be treated by a caregiver if they don't belong to his working areas.

Due to the intrinsic nature of the care demand, real-time optimization of operations have been considered. Thus, Du et al. [13] have established an effective real-time scheduling model to minimize the total required time of scheduling, and proposed an improved memetic algorithm to optimize the model. Their work aims at resolving the real-time scheduling problem considering the unexpected events in home health care. The unexpected events are classified into two categories: the insertion of emergency patients and the temporary cancelation of medical service. 
Alternatives to provide a robust planning have also been explored. In order to face possible uncertainties regarding the optimization model, Shi et al. [28] have formulated a robust model for an home health care routing and scheduling problem by taking into account uncertain travel and service times. Specifically, the non-deterministic variables are defined based on the theory of budget uncertainty, and then the arrival time of each caregiver is rewritten as a complicated recursive function. Their experimental results from Monte Carlo simulation highlight the strength of considering uncertainties when modeling the problem. Similarly, uncertainties have also been considered regarding the unknown demand in care and spatial dimensions, combination of skills to cover a care and individual trips visiting patients. In this regard, Rodriguez et al. [27] have aimed at calculating the amount of personnel required to ensure the activity of a home health care center on a tactical horizon. The authors have proposed a two-stage approach based on integer linear stochastic programming, which exploits historical medical data. The first stage calculates (near-)optimal levels of resources for possible demand scenarios, while the second stage computes the optimal number of caregiver for each profession to meet a target coverage indicator.

Some existing works focus on the assignment of caregivers to patients, but only in the case of a single home health care office. Robust approaches have been considered by Lanzarone and Matta [21] for the assignment of a reference caregiver to each assisted patient, under continuity of care. Furthermore, Errarhout et al. [15] have studied the assignment problem related to the home health care structures. They assign nurses to a deterministic number of patients and care in order to balance the nurses' workload. However, the focus of the paper is only on the assignment problem and no attention is paid to aspects of routing and scheduling. Furthermore, a single home health care office is considered. Later on, Yalçındağ et al. [33] have also studied the patient assignment problem in home health care. The assignment of visits to caregivers is performed in order to balance the trade-off between workload balancing and total travel time minimization. However, only a single home health care office is considered in this work.

In addition, Trautsamwieser et al. [31] have also considered that nurses may not only start from the home health care organization but also from their own home. In this situation, they are paid for the driving time from their home to the first patient. However, we have not considered this possibility since caregivers use vehicles provided by the company, which corresponds to most real cases.

Among the literature considering multiple depot where caregivers must start and finish their workday at the same home health care office, Bard et al. [2] solve the weekly therapist scheduling problem and present a series of models including single and multiple home bases. They consider that each therapist has a unique location that is referred to as the home base where the therapist starts and ends the day. Similarly, Decerle et al. [10] have modeled the home health care problem with multiple depots where caregivers are assigned to one of them. They use a memetic algorithm on both single and multi-depot home health care problem with soft time window and synchronization constraints.

However, the upstream assignment of caregivers to a cluster may lead the resolution of the problem to a local optimum. Moreover, in the case of home health care structures with multiple offices, there is, to the best of our knowledge, no existing study from the literature which allows caregivers and patients to be dynamically assigned to a home health care office. Freely assigning the caregivers to offices during the resolution of the problem may lead to better results by enabling solutions that previously would have been considered impossible. In this way, caregivers can be assigned optimally to offices in order to obtain better scheduling and reduce their traveling times. On a daily basis, some caregivers may be pre-assigned to one of the health care office to avoid a complete reorder. In addition, this approach can be used periodically to readjust the allocation of staff to health care offices when the number of staff varies or if the geographical location of patients is changed, for example by taking responsibility for new geographical areas.

To the best of our knowledge, there is no work devoted to the multi-depot home health care assignment, routing and scheduling problem without prior assignment of caregivers to the home health care offices. More specifically, the contributions of the current work are as follows. First, we propose a mixed-integer linear programming formulation of the multi-depot home health care problem without prior assignment of caregivers to the home health care offices. Secondly, a matheuristic-based approach is proposed in different versions, to tackle the 
proposed problem, and find the most efficient version. Finally, performance of the matheuristic-based approach are evaluated by applying the algorithm on problem instances of varying number of visits, caregivers, home health care offices and synchronized visits. Results are compared with best solutions obtained by a commercial solver, Gurobi, and with a lower bound computed using a Lagrangian relaxation of the problem.

This paper is outlined as follows. In the next section, the characteristics of the studied system are presented. In Section 3, the mixed-integer programming formulation of the multi-depot home health care problem without prior assignment of caregivers to the home health care offices is detailed. Section 4 contains the description of the matheuristic approach for the resolution of the problem, including, different assignment strategies of visits to the home health care offices. In Section 5, we develop a lower bound on the optimal solution of the precise model based on the Lagrangian relaxation method. The test instances and results of the experiments are presented in Section 6. Finally, some concluding remarks, as well as some perspectives on future works, are drawn in the last section.

\section{System DESCRIPTION}

The multi-depot home health care problem is defined as follows.

Given a set of caregivers, a set of home health care offices and a set of visits to be performed at the patients' location, the goal is to find a valid schedule and a route on a one-day period for each caregiver. The resulting planning has to indicate which visit has to be performed by which caregiver and when the visit should start. Each caregiver has a defined working time window which means they work only during this time period. Caregivers may or may not be assigned to a home health care office. If a caregiver is assigned to an office, it means that they have to start and finish his working day at his assigned office. On the other hand, if a caregiver is not preassigned to any office prior to the resolution of the routing and scheduling problem, then they will be assigned to an office freely during the resolution, depending on the solution providing the best result.

Each caregiver uses the same transportation mode (i.e. a car provided by the home health care company). Working overtime is not allowed due to an increased cost of work for the company. Home health care caregivers are either nurses or auxiliary nurses.

The time at which a caregiver has to start a visit is restricted by a time window. Consequently, the patient and the home health care company agree on a time window in which the care has to start. Patient time window are considered hard time window, which means no advance or delay is allowed. Each patient time window is specific for each visit depending on the patient availability or the type of care to provide. Similarly as the caregivers, the visits can be assigned to a home health care office. In that case, the visit should be performed by a caregiver assigned to the same home health care office as the visit. Furthermore, the assignment of a visit to a home health care office is not mandatory.

The visits are carried out by only one category of caregiver (e.g. an injection is performed only by a nurse). Therefore some visit/caregiver combinations are invalid. Some visits have to be synchronized when the health care has to be performed by two caregivers simultaneously. Therefore, the arrival time of both caregivers must be equal in the case of synchronized visits.

Finally, we assume that the main objective of the home health care company is to minimize the total time traveled by the caregivers during their working day.

\section{MODEL DESCRIPTION}

In this section, the mixed integer programming formulation of the multi-depot home health care problem is presented.

\subsection{Notation}

The multi-depot home health care routing and scheduling problem is modeled on a graph $G=(N, A)$ where $N$ is the set of nodes and $A$ the set of arcs. The set of visits to perform is denoted $O$ and the set of home health 
care offices is denoted $P$. Thus, $N=O \cup P$. Each visit is represented by a separated node in the graph, whether two or more visits are associated with the same physical location or not. For example, if a given patient requires two visits during the day, a node will be created for each visit and they will both have the same geographical location. Using this information, the set of arcs is defined as $A=\{(i, j) \mid i, j \in N, i \neq j\}$. Each $\operatorname{arc}(i, j) \in A$ has a distance $d_{i j}$.

The set of caregivers is denoted $S$. For each caregiver $i \in S$, a working hard time window $\left[\alpha_{i}, \beta_{i}\right]$ is known. The set $R=\left\{r_{1}=\mathcal{N}, r_{2}=\mathcal{U}\right\}$ represents the possible qualifications that can have caregivers. Nurses have the job role $\mathcal{N}$ and the auxiliary nurses have the job role $\mathcal{U}$. The association of a job role to a staff member $i$ is defined by the binary parameter $\omega_{i j}$. The parameter $\omega_{i j}$ is equal to 1 if the staff member $i$ has the role $r_{j}$ and 0 otherwise.

The home health care offices are located in different places and therefore have different locations. The binary parameter $\gamma_{i}^{k}=1$ if the caregiver $i$ is assigned to the home health care office $k$, and zero otherwise.

Moreover, for each visit $i \in O$ at the patient's home, a duration $c_{i}$ represents the time needed to perform the care. A hard time window $\left[a_{i}, b_{i}\right]$ represents the availability of the patient to receive a care. The binary parameter $\delta_{i j}=1$ if the visits $i$ and $j$ are synchronized, and 0 otherwise.

In addition, the binary parameter $\phi_{i}^{j}=1$ indicates that the patient $i$ is assigned to the home health care office $j$, and zero otherwise. Finally, the caregiver qualification needed to perform the job $i$ is defined by $\rho_{i j}$. If the visit requires a caregiver with the job role $r_{j}, \rho_{i j}=1$, and 0 otherwise.

We consider a planning period of a single day.

In order to formulate the home health care problem, we use several decision variables. The decision variable $x_{i j}^{k}=1$ if the caregiver $k$ travels from $i$ to $j$, and zero otherwise. The variable $t_{i}$ defines the starting time of the visit $i$. Moreover, the variable $\eta_{i}^{k}=1$ if the caregiver $k$ is assigned to the home health care office $i$, and zero otherwise.

Furthermore, considering a caregiver $i$ and a home health care office $k$, if $\gamma_{i}^{k}=1$, then $\eta_{i}^{k}=1$. However, if $\gamma_{i}^{k}=0$, then $\eta_{i}^{k}$ could be equal to either 0 or 1 .

Finally, it should be highlighted that the proposed model allows partial or complete assignment of caregivers and visits to the home health care office. Therefore, it is possible to solve the problem without assigning any caregivers or visits to the home health care offices to obtain an optimal solution. In addition, the model can be reduced to the current state-of-art methods from the literature where all caregivers are assigned to the home health care offices before the resolution of the model. Compared with the state of the art, this variant brings more flexibility to the problem modeling. In the meantime, it should be noted that when no prior assignment of caregivers and visits is made, the complexity of the problem increases as caregivers can begin their route in any home care office, resulting in a higher number of variable values to resolve. However, the progressive assignment of caregivers and visits in the experiments is intended to reduce the complexity of the problem, and thus reduce the calculation time.

In terms of the growth of variables, the complexity of the problem grows exponentially as the number of caregivers, visits or depots increases.

\subsection{Problem formulation}

In this part, the formulation of the problem is presented.

In order to ease the understanding of the mathematical model, a description of the sets, parameters and variables used is detailed in Table 1.

The multi-depot home health care problem is formulated as follows:

$$
\min \sum_{i \in N} \sum_{j \in N} \sum_{k \in S} x_{i j}^{k} \times d_{i j}
$$


TABLE 1. Description of the sets, parameters and variables used in the mathematical model.

\begin{tabular}{lll}
\hline \hline & Type & Description \\
\hline Sets & & \\
\hline$N$ & & Set of nodes \\
$S$ & & Set of caregivers \\
$O$ & Set of visits \\
$P$ & Set of home health care offices \\
$R$ & & Set of caregivers' qualification \\
\hline Parameters & & \\
\hline$d_{i j}$ & Positive real & Travel time between the nodes $i$ and $j$ \\
$c_{i}$ & Positive real & Duration of the visit $i$ \\
$\phi_{i}^{j}$ & Binary & 1 if patient $i$ is assigned to home health care office $j, 0$ otherwise \\
$\gamma_{i}^{k}$ & Binary & 1 if caregiver $i$ is assigned to home health care office $k, 0$ otherwise \\
{$\left[\alpha_{i}, \beta_{i}\right]$} & Positive real interval & Working time window of caregiver $i$ \\
{$\left[a_{i}, b_{i}\right]$} & Positive real interval & Availability time window of visit $i$ \\
$\delta_{i j}$ & Binary & 1 if visits $i$ and $j$ are synchronized, 0 otherwise \\
$\omega_{i}$ & R & Qualification of the caregiver $i$ \\
$\rho_{i}$ & R & Qualification required to perform the visit $i$ \\
\hline Variables & & \\
\hline$\eta_{i}^{k}$ & Binary & 1 if caregiver $i$ is assigned to home health care office $k, 0$ otherwise \\
$x_{i j}^{k}$ & Binary & 1 if caregiver $k$ travels from $i$ to $j, 0$ otherwise \\
$t_{i}$ & Positive real & Starting time of the visit $i$ \\
\hline
\end{tabular}

subject to:

$$
\begin{aligned}
& \sum_{j \in N} \sum_{k \in S} x_{i j}^{k}=1 \\
& \sum_{j \in O} x_{i j}^{k} \geq \gamma_{k}^{i} \\
& \sum_{j \in O} x_{j i}^{k} \geq \gamma_{k}^{i} \\
& \sum_{i \in P} \sum_{j \in O} x_{j i}^{k}=1 \\
& \sum_{j \in O} x_{j i}^{k}=\sum_{j \in O} x_{i j}^{k} \\
& \sum_{\substack{j \in N \\
i \neq j}} x_{i j}^{k}=\sum_{\substack{j \in N \\
i \neq j}} x_{j i}^{k} \\
& \eta_{i}^{k}=\sum_{\substack{j \in N \\
j \neq i}} x_{i j}^{k} \\
& \sum_{l \in N} x_{i l}^{k} \leq \eta_{j}^{k} \\
& a_{i} \leq t_{i} \\
& b_{i} \geq t_{i}
\end{aligned}
$$$$
\forall i \in O
$$$$
\forall i \in P, \quad k \in S
$$$$
\forall i \in P, \quad k \in S
$$$$
\forall k \in S
$$$$
\forall i \in P, \quad k \in S
$$$$
\forall i \in N, \quad k \in S
$$$$
\forall i \in P, \quad k \in S
$$$$
\forall i \in O, \quad j \in P, \quad k \in S, \quad \phi_{i}^{j}=1
$$$$
\forall i \in O
$$$$
\forall i \in O
$$ 


$$
\begin{array}{lll}
t_{j} \geq t_{i}+c_{i}+d_{i j}+\left(\sum_{k \in S} x_{i j}^{k}-1\right) \times M & \forall i \in N, \quad j \in O, \quad i \neq j \\
\beta_{k} \geq t_{i}+\left(c_{i}+d_{i j}\right) \times\left(\eta_{j}^{k}\right)+\left(x_{i j}^{k}-1\right) \times M & \forall i \in O, \quad j \in P, \quad k \in S \\
t_{i} \geq\left(\alpha_{k}+d_{i j}\right) \times\left(\eta_{j}^{k}\right)+\left(x_{j i}^{k}-1\right) \times M & \forall i \in O, \quad j \in P, \quad k \in S \\
t_{i}=t_{j} & \forall i \in O, \quad j \in O, \quad i \neq j, \quad \delta_{i j}=1 \\
\sum_{\substack{j \in N \\
i \neq j}} x_{j i}^{k}=0 & \forall i \in O, \quad k \in S, \quad \omega_{k} \neq \rho_{i} \\
x_{i j}^{k} \in\{0,1\} & \\
\eta_{i}^{k} \in\{0,1\} & \forall i \in N, \quad j \in N, \quad k \in S \\
t_{i} \in \mathbb{R}^{+} & \forall i \in P, \quad k \in S
\end{array}
$$

The objective function (3.1) minimizes the total traveling time of the caregivers.

Constraint (3.2) ensures that a visit is done by only one caregiver. Caregivers' departure and arrival to their home health care office, to which they might be associated, is ensured by the constraints (3.3) and (3.4). The constraint (3.5) verifies that caregivers come back to a home care office. In the same way, the constraint (3.6) verifies that the caregivers return to the same home health care office as the one from which they left. The flow conservation is guaranteed by the constraint (3.7).

The assignment of a caregiver to a home health care office is verified by the constraint (3.8) while the association of a patient with a home health care office is ensured by the constraint (3.9).

The constraints (3.10) and (3.11) make sure that the care starts in the time window of patient availability.

Constraint (3.12) make sure that the caregivers have enough time between two visits to perform the first and then go to the next patient. In addition, the constraints (3.13) and (3.14) ensure that the caregivers work only during the opening period of the home health care office from which they started their workday.

Synchronization of visits is ensured by the constraint (3.15) that allows both visits to start at the same time.

The constraint (3.16) verifies that the caregivers have the necessary qualification to perform the care.

Finally, the constraint (3.17) defines the domain of the variables.

\section{Matheuristic-BASED APPROACH}

In this section, we present a matheuristic-based approach for the resolution of the multi-depot home health care problem. First, the assignment of visits and caregivers (i.e. nurses and auxiliary nurses) to the home health care offices is performed. Subsequently, the routing and scheduling steps are solved using a two-phases matheuristic suggested by Decerle et al. [9].

Matheuristic are algorithms made by the interoperation of heuristics and mathematical programming techniques.

In order to give an overview of the structure of the algorithm, Figure 1 represents the flow chart of the matheuristic-based approach. Both steps of route planning for nurses and auxiliary nurses are solved with the mathematical programming model.

Each step of the algorithm is detailed here after.

The main inputs of the matheuristic algorithm are the parameters of the mathematical model, the percentage of visits and caregivers to be pre-assigned, and the strategy used for assigning visits, as described in the next section. 


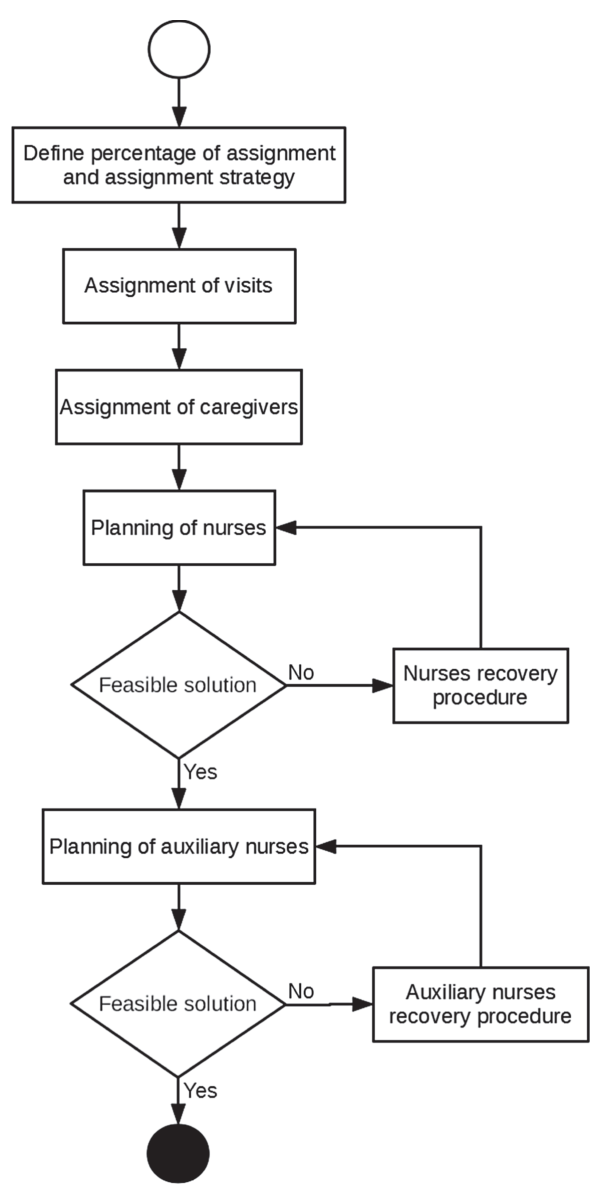

FIGURE 1. Structure of the matheuristic-based approach.

\subsection{Assignment of visits}

The aim of this step is to perform the prior assignment of some visits to the home health care offices where they will most likely receive the care of a caregiver from the assigned home health care office. Being assigned to a home health care office for a visit means that the care will be performed by a caregiver who started his route to the assigned home health care office. In order to perform the assignment of visits to the home health care offices, we consider the set of visits $O$ and the set of home health care offices $P$.

In this section, various assignment strategies are presented in order to identify the most effective one.

For each unassigned visit $i \in O$, we have $\sum_{j \in P} \phi_{i}^{j}=0$. In addition, the assignment cost of the visit $i$ is represented by $\mu_{i}$. Unassigned visits with the lowest assignment cost will be assigned first.

The assignment cost is computed as follows in each configuration:

Nearest Depot (ND). The assignment cost of the visit $i$ is the traveling time between the visit $i$ and its closest office. In this case, the assignment cost is:

$$
\mu_{i}=\min \left(d_{i j}\right) \quad \forall i \in O, \quad j \in P .
$$

Isolated Nearest Depot (IND). The assignment cost of the visit $i$ is the ratio between the traveling time between the visit $i$ and its closest office and the traveling time between the visit $i$ and its second closest office. 
Consequently, we consider:

$$
\mu_{i}=\frac{\min \left(d_{i j}\right)}{\min \left(d_{i j} \backslash\left\{\min \left(d_{i j}\right)\right\}\right)} \quad \forall i \in O, \quad j \in P .
$$

Average Nearest Cluster (ANC). The assignment cost of the visit $i$ is defined as the lowest average traveling time between the visit $i$ and a cluster related to a home health care office. The cluster $C_{j}$ contains the home health care office $j$ itself as well as all the visits already assigned to it. We define the set

$$
C_{j}=\left\{i \in O / \phi_{i}^{j}=1\right\} \cup\{j\} \quad \forall j \in P .
$$

The value of $C_{j}$ is updated after each newly made assignment. Subsequently, the average traveling time between the visit $i$ and the cluster $C_{j}$ denoted as $\theta_{i}^{j}$ is the average traveling time between the visit $i$ and each node contained in the cluster $C_{j}$.

$$
\theta_{i}^{j}=\frac{1}{\left|C_{j}\right|} \times \sum_{k \in C_{j}} d_{i k} \quad \forall i \in O, \quad j \in P .
$$

As a result, the assignment cost is defined as:

$$
\mu_{i}=\min \left(\theta_{i}^{j}\right) \quad \forall i \in O, \quad j \in P .
$$

Average Nearest Isolated Cluster (ANIC). The assignment cost of the visit $i$ is defined as the ratio between the lowest average traveling time between the visit $i$ and a cluster related to a home health care office and the second lowest average traveling time between the visit $i$ and a cluster related to another home health care office. As a result, we consider the assignment cost as:

$$
\mu_{i}=\frac{\min \left(\theta_{i}^{j}\right)}{\min \left(\theta_{i}^{j} \backslash\left\{\min \left(\theta_{i}^{j}\right)\right\}\right)} \quad \forall i \in O, \quad j \in P .
$$

Nearest Cluster Node (NCN). The assignment cost of the visit $i$ is defined as the shortest traveling time between the visit $i$ and a node belonging to a cluster related to a home health care office. $\sigma_{i}^{j}$ corresponds to the shortest traveling time between the visit $i$ and any node contained in the cluster $C_{j}$.

$$
\sigma_{i}^{j}=\min \left(d_{i k}\right) \quad \forall i \in O, \quad j \in P, \quad k \in C_{j} .
$$

In this case, the assignment cost is considered as:

$$
\mu_{i}=\min \left(\sigma_{i}^{j}\right) \quad i \in O, \quad j \in P .
$$

Nearest Isolated Cluster Node (NICN). The assignment cost of the visit $i$ is defined as the ratio between the shortest traveling time between the visit $i$ and a node belonging to a cluster related to a home health care office and the second shortest traveling time between the visit $i$ and a node belonging to a cluster related to another home health care office. In this case, the assignment cost is:

$$
\mu_{i}=\frac{\min \left(\sigma_{i}^{j}\right)}{\min \left(\sigma_{i}^{j} \backslash\left\{\min \left(\sigma_{i}^{j}\right)\right\}\right)} \quad \forall i \in O, \quad j \in P .
$$

Unassigned visits with the lowest cost $\mu_{i}$ are assigned first, to their closest depot, depending on the assignment strategy applied. 


\section{A}

B

D
E
2

C

Figure 2. Geographical location of 2 home health care offices and 5 visits.

\section{A}

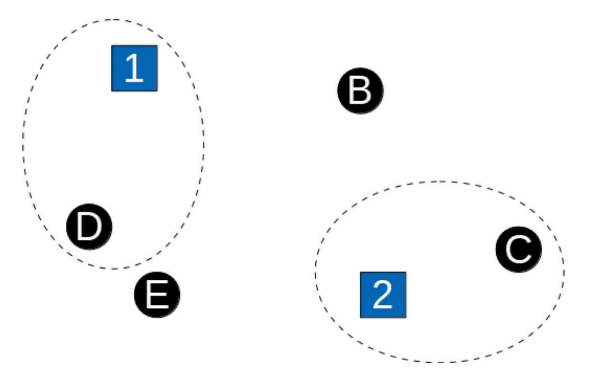

Figure 3. Assignment of the visits D and $\mathrm{C}$ to the home health care offices 1 and 2.

TABLE 2. Assignment ratio computation of the visits.

\begin{tabular}{llll}
\hline & Office 1 & Office 2 & Assignment ratio \\
\hline Visit A & 1.84 & 3.30 & 0.56 \\
Visit B & 2.01 & 1.81 & 0.90 \\
Visit C & 3.76 & 1.27 & 0.34 \\
Visit D & 1.46 & 2.67 & 0.55 \\
Visit E & 2.01 & 2 & 1 \\
\hline
\end{tabular}

In versions $I N D, A N I C$ and $N I C N$, visits which are relatively close to a depot and far from others have a higher assignment priority.

The assignment process is repeated until the required number of visits is assigned.

Consider an example with five visits and two home health care offices as presented in Figure 2. The aim is to assign up to $50 \%$ of visits using the version Isolated Nearest Depot (IND) as an assignment strategy. In Figures 2 and 3, visits are represented as circles and offices as squares.

The travel time between the 5 visits' location and the 2 home health care offices, and the assignment ratio $\mu_{i}$ are computed in Table 2.

The visit $\mathrm{C}$ is assigned to the home health care office 2 , since it has the lowest assignment ratio and its closest office is the depot 2. At this point, one out of five (i.e. 20\%) visits is assigned. The visit $\mathrm{C}$ is removed from the list of unassigned visits. Then, the lowest assignment ratio among the unassigned visits is related to the visit 
D. Consequently, visit D is assigned to the office 1 and removed from the list of unassigned visits. Since two out of five (i.e. 40\%) visits are assigned, assigning a new visit would exceed the $50 \%$ limit of assigned visits. Therefore, the assignment of visits to offices stops with 2 assigned visits (see Fig. 3).

\subsection{Assignment of caregivers}

After assigning the visits, the caregivers (i.e. nurses and auxiliary nurses) are also assigned to a home health care office. Being assigned to a home health care office for a caregiver means that the caregiver must start and end his workday at his assigned home health care office.

The number of caregivers assigned is proportional to the number of visits assigned. Furthermore, we take into account the level of qualification required for the care of assigned visits. For example, if $50 \%$ of visits is assigned to a home health care office with $70 \%$ of the assigned visits requiring a nurse and $30 \%$ an auxiliary nurse, then $35 \%$ of available nurses and $15 \%$ of available auxiliary nurses will be assigned.

In addition, the assignment of caregivers to the home health care offices ensures that all home care offices will have at least one caregiver with the required qualification from visits assigned to the same home care office. Therefore, if some visits require nurses and auxiliary nurses are assigned to a home health care office, then at least one nurse and one auxiliary nurse will be assigned to the home health care office. In addition, caregivers are assigned to the home health care offices proportionally as the number of visits assigned to each office.

These assignment steps may improve the speed of resolution of the next step by imposing the value of certain variables and thus by limiting the size of the problem.

\subsection{Routing and scheduling}

In this part, the assignment of visits and caregivers to the home health care offices has been performed. As a result, the focus is on routing and scheduling of caregivers.

The route planning of the nurses and auxiliary nurses ensures that patients' time window are respected, according to constraints (3.10) and (3.11).

At this stage, a part of the visits and caregivers have been assigned to a home health care office. The rest of the visits and caregivers who have not been assigned to any home health care office will be freely assigned to any home health care office, depending on the best solution found by the matheuristic.

In order to define the route and the schedule of each caregiver, we reuse the two-phases matheuristic suggested by Decerle et al. [9]. Indeed, the two-phases matheuristic provides good results with a small optimal gap and a short computer time on instances of the home health care problem with various levels of caregivers' qualification.

In the first phase, the resolution of the model is performed only on the nurses. Consequently, after the first phase of resolution, the planning of all the nurses is defined.

To summarize, the inputs of the first phase of resolution are:

- The assignment of nurses to their associated home health care offices.

- The assignment of visits requiring a nurse to their associated home health care offices.

- All the visits requiring a nurse to perform it.

- All the home care offices contained in the set $P$.

- All the nurses contained in the set $S$.

As a result, we obtain a planning for each nurse as well as the assignment of each nurse to a home health care office. The assignment of nurses to their home health care office does not change after the resolution.

Then, the second phase performs the resolution of the model concerning the auxiliary nurses, by taking into account the planning obtained after the first phase.

In order to perform the resolution, the inputs of the second phase are:

- The planning of the nurses computed in the first phase.

- The assignment of auxiliary nurses to their associated home health care offices.

- The assignment of visits requiring an auxiliary nurse to their associated home health care offices. 
- All the visits of the set $O$.

- All the home care offices contained in the set $P$.

- All the caregivers contained in the set $S$.

Therefore, the planning obtained at the end of the second phase defines the route and schedule of each caregiver. Furthermore, the assignment of caregivers and patients performed previously has not been altered and has been conserved in the final planning.

\subsection{Improvement in the feasibility of solutions}

The two-phases matheuristic from the literature for routing and scheduling presents a major inconvenient. In some cases, it might be impossible to find a feasible solution when the planning of the nurses has been performed. Indeed, due to the synchronization constraint, the planning of the auxiliary nurses is linked to the nurses' planning. Depending on the solution obtained during the first phase of the resolution, it might be impossible to obtain a valid schedule for the auxiliary nurses, respecting the synchronization constraint (arrival time of the nurse and auxiliary nurse must be equal), leading to the impossibility to solve the problem.

As a result, several improvements to the initial two-phases matheuristic are proposed in order to overcome this issue.

After the planning of the nurses, no feasible solution may be found because of the former assignment of visits and caregivers to the home health care offices. In this case, a "Nurses recovery procedure" is performed until a feasible solution can be found for the planning of the nurses. The recovery procedure consists of the following steps:

- The assignment of a nurse to a home health care office is removed.

- The assignment of a visit requiring a nurse to a home health care office is removed.

At the beginning of the second phase, if no feasible solution can be obtained to perform the final planning, the "Auxiliary nurses recovery procedure" including several actions on the nurses planning obtained after the first phase are repeated until a feasible solution is found:

- A randomly selected route of a nurse is removed. All the visits of the route are not scheduled anymore. In addition, if some of the visits were assigned to a home health care office, the assignment is removed. In addition, if the nurse who was related to the destroyed route was assigned to a home health care office, the assignment of the nurse to the office is also removed.

- The assignment of an auxiliary nurse to a home health care office is removed.

- The assignment of a visit requiring an auxiliary nurse to a home health care office is removed.

These actions are repeated until the resolution of the second phase leads to a feasible solution.

Finally, thanks to this improvement procedure, the matheuristic is now able to find a feasible planning in all cases.

\section{LAGRANGIAN LOWER BOUND DEVIATION}

\subsection{Lagrangian relaxation}

The Lagrangian relaxation (LR) is a widely used technique in combinatorial optimization introduced by Fisher [18], where an original difficult problem is approximated by a simpler problem. The basic idea is to relax the complicating constraints into the objective function and penalize violations of the constraints using Lagrangian multipliers. This technique has been successfully applied to other variants of the home health care problem by Chen et al. [6] and Bard and Purnomo [1].

Similarly to the m-traveling salesman problem with time windows studied by Desrosiers et al. [11], we decide to relax the constraint on visiting each node (3.2) since it provides the best results on this type of problem. 
Consequently, by introducing the non-negative Lagrangian multipliers $\left\{\lambda_{i}\right\}$, the Lagrangian relaxation of the constraint (3.2) generates the following relaxed problem:

$$
Z(\lambda)=\left(\min \left(\sum_{i \in N} \sum_{j \in N} \sum_{k \in S} x_{i j}^{k} \times d_{i j}+\sum_{i \in O} \lambda_{i}\left(1-\sum_{j \in N} \sum_{k \in S} x_{i j}^{k}\right)\right)\right)
$$

subject to constraints (3.3) to (3.16).

Here $\lambda$ is a vector of non-negative Lagrangian multipliers with elements $\left\{\lambda_{i}\right\}$, where $i=1,2, \ldots|O|$.

The aim of solving the Lagrangian dual is to find a lower bound on the optimal value of our original optimization problem. Therefore, the Lagrangian dual is defined as:

$$
Z_{D}=\max \left(Z(\lambda)_{\lambda \geq 0}\right) .
$$

\subsection{Updating the Lagrangian multipliers}

After relaxing the original problem, we can iteratively update the Lagrangian multipliers and solve the Lagrangian dual problem through subgradient optimisation. At each iteration, subproblems are solved given the Lagrangian multipliers $\lambda^{t}$.

Initially, we have $t=0$ and $\lambda_{i}^{t}=0$. Then at each iteration, $\lambda$ can be updated by $\lambda_{i}^{t+1}=\max \left\{0, \lambda_{i}^{t}+\gamma^{t} f_{i}^{t}\right\}$, where $\gamma^{t}$ is the step size at the $t^{t h}$ iteration and $f_{i}^{t}$ is the subgradient of the dual problem. The subgradient component can be computed by the relaxed constraint (3.2), i.e. $f_{i}^{t}=1-\sum_{j \in N} \sum_{k \in S} x_{i j}^{k}$.

The definition of the stepsize $\gamma$ is of importance since the speed of convergence depends heavily on the stepsize. Following the formula proposed by Held et al. [20] for adapting the stepsize, the step size $\gamma^{t}$ is given by:

$$
\gamma^{t}=\mu^{t} \times \frac{Z^{*}-Z\left(\lambda^{t}\right)}{\sum_{i \in O}\left(f_{i}^{t}\right)^{2}}
$$

where:

- $Z^{*}$ is the value of the best solution for the original problem found so far

$-\mu^{t}$ is a decreasing adaption parameter with $0<\mu^{0} \leq 2$ and

$$
\mu^{t+1}=\left\{\begin{array}{l}
\alpha \mu^{t}, \quad \text { if } Z_{D} \text { did not increase in } \\
\text { the last } T \text { iterations } \\
\mu^{t}, \quad \text { otherwise }
\end{array}\right.
$$

with parameters $0<\alpha<1$ and $T>1$.

Finally, the algorithm stops either if $f_{i}^{t}=0$ because the optimal value has been reached, if the time limit of two hours have been reached or if the improvement between two consecutive iterations is equal to or less than a certain value, which is set to 0.001 in this paper. This value has been chosen in such a way that it represents only an infinitesimal improvement (less than one second) in the quality of the solution obtained, i.e. the planning of the caregivers.

\section{NUMERICAL EXPERIMENTS}

In order to evaluate the efficiency of the suggested matheuristic, a set of experiments is performed on various test instances.

It should be emphasized that the comparison with the existing results of the literature is impossible. Indeed, no work in the literature has addressed the problem by taking into account the same constraints, in particular without prior assignment of caregivers to home care offices. 
TABle 3. Characteristics of the test instances. The lines are: the number of visits $|O|$, the number of synchronization $\mid$ Sync $\mid$, the number of home health care offices $|P|$.

\begin{tabular}{lll}
\hline \hline & Small size & Medium-sized \\
\hline$|O|$ & 25 & 50 \\
$\mid$ Sync $\mid$ & 2 & 5 \\
$|P|$ & 2 & 3 \\
\hline
\end{tabular}

\subsection{Instances and settings of experiments}

Test instances have been generated based on the benchmark instances proposed by Solomon [29] for the vehicle routing problem with time window (VRPTW). The benchmark contains 56 instances whose patients' location are either randomly generated, clustered or a mix of random and clustered. In addition, instances consider either 25 or 50 visits to perform.

However, all of the initial instances contain only one depot, no synchronization between visits and no qualification levels. Therefore, original instances have been altered in order to introduce multiple depots, synchronized visits and qualification level required to perform each visit. To summarize, the characteristics of the test instances used for the experiments is presented in Table 3. As well, the number of caregivers required to provide care varies and depends on each of the 56 instances.

The various assignment strategies presented in Section 4.1 for assigning visits to the home health care offices are experimented in order to compare their performance. Furthermore, for each assignment strategy, the matheuristic is tested twice. Indeed, the two-phases matheuristic suggested by Decerle et al. [9] for the routing and scheduling problem is experimented with or without the technical improvements detailed in Section 4.3 to avoid unfeasible solutions.

In addition, the percentage of visits and caregivers assigned to the home health care offices varies in order to study the impact on the quality of the solution obtained as well as the computation time. Consequently, the proportion of assigned visits and caregivers varies from 0 to $100 \%$ by step of $10 \%$.

Concerning the tuning of the Lagrangian relaxation, the parameter $\mu$ is initialized to a value of 1.2 (i.e. $\mu^{0}=1.2$ ) and halved (i.e. $\left.\alpha=0.5\right)$ if the value of $Z_{D}$ did not increase in the last two iterations (i.e. $T=2$ ). These values have been chosen empirically since the lower bound obtained is close to the optimal solution for most instances.

Concerning the computation time, the two phases of the matheuristic for the routing and scheduling phases to resolve nurses' planning and then auxiliary nurses' planning are both resolved using [19] (version 6.0.5) with a time limit of $60 \mathrm{~min}$ each, that to say $120 \mathrm{~min}$ at most. In contrast, the results provided by the matheuristic are compared with a global optimization of the problem using Gurobi with a time limit of 120 min, and with the lower bound computed by the Lagrangian relaxation. Since the algorithm makes decisions at both the tactical and operational levels, the two-hour limit was chosen to give the mathematical solver enough time to obtain a good solution.

The matheuristic-based approach is evaluated once for each configuration since it will always produce the same result for each instance. Thus, there is no need to repeat the experiments several times.

Finally, the experiments have been performed on a desktop with an Intel (R) Xeon (R) CPU E5-2630 v3 @ $2.40 \mathrm{GHz}$ with $32 \mathrm{~Gb}$ of RAM memory.

\subsection{Computational results}

In this section, the matheuristic-based approach is compared to the results obtained by the optimal solving of the problem using an optimization solver, and with a lower bound obtained by a Lagrangian relaxation of the problem. 
First, the results obtained by the matheuristic-based approach are compared with the results obtained by the optimization solver Gurobi on small size and middle-sized instances in order to validate the efficiency of the matheuristic-based approach. Then, the lower bound obtained by Lagrangian relaxation are compared with the best-found solution for each instance and with the lower bound obtained by Gurobi. The aim is to assess the quality of the lower bound obtained by Lagrangian relaxation. Finally, the results of the matheuristic-based approach are compared with the lower bound obtained by Lagrangian relaxation on middle-sized instances, where Gurobi cannot find the optimal solution for many instances.

The column "Solving method" indicates which method has been used to perform the experiments. The values are either "MIP" or "Matheuristic". The line "MIP" indicates the results obtained by the optimization solver Gurobi on the original problem. The results of the matheuristic are presented under the name "Matheuristic", followed by the name of the assignment strategy applied for the assignment of visits to the home health care offices.

The column "Visits assigned (\%)" indicates the percentage of visits and caregivers that have been assigned to the home health care offices during the assignment step, prior to the routing and scheduling.

The column "Deviation ratio (\%)" represents the gap obtained by the matheuristic compared to the optimization solver Gurobi. The lower the deviation ratio is and the better the result is. Consequently, the deviation ratio is computed as follows:

$$
\text { Deviation ratio }=\frac{\text { Matheuristic }- \text { MIP }}{\text { MIP }}
$$

Moreover, the column "Solving time (s)" indicates the computational time needed by the algorithm in seconds. Finally, the column "Feasible solution (\%)" indicates the percentage of instances where a feasible solution has been found. The percentage is computed regarding the number of feasible solution found over the 56 test instances.

In addition, the sub-columns "Original" and "Improved" respectively indicate whether the approach used for the routing and scheduling is the original matheuristic suggested by Decerle et al. [9] or the original matheuristic with the improvements presented in Section 4.3 to always obtain a feasible solution.

It should be noted that each line of results in Tables 4 and 5 is an average of the results obtained in the 56 instances.

Moreover, the optimization solver has found the optimal solution to the multi-depot home health care problem in 55 out 56 instances $(98.21 \%$ ) with 25 patients and 28 out of 56 instances (50\%) with 50 patients.

First, the results obtained by the matheuristic-based approach are compared with the results obtained by the optimization solver Gurobi on small size and middle-sized instances.

Based on the results provided by the Tables 4 and 5, the matheuristic-based approach proves to be very efficient compared to the optimization solver.

As a matter of fact, the matheuristic-based approach maintains a low deviation ratio compared to the results obtained by the optimization solver Gurobi.

Regardless of the assignment strategy used for small size instances, the deviation ratio does not exceed $10 \%$ with Gurobi when up to $80 \%$ of visits are pre-assigned to the home health care offices (except for the first assignment strategy "Nearest Depot"). In addition, these results are noticeable since the matheuristic-based approach performance is compared in most cases with the optimal solution obtained by the optimization solver. Lastly, it should be noted that two assignment strategies seem to detach from others by providing much better results: "Average Nearest Cluster" and "Nearest Cluster Node". Indeed, the deviation ratio increases much more slowly even when a high proportion of visits and caregivers are assigned to the home health care offices.

Another criterion to compare is the solving time of each method. Furthermore, the matheuristic-based approach is much faster than the optimization solver. As an example, the average solving time of the optimization solver over the 56 small size instances is $352.28 \mathrm{~s}$ while it stays around only one second for the matheuristic-based approach. 
TABLE 4. Comparison of the results of the matheuristic-based approach with the commercial optimization solver on small size instances.

\begin{tabular}{|c|c|c|c|c|c|c|c|}
\hline \multirow[t]{2}{*}{ Solving method } & \multirow{2}{*}{$\begin{array}{l}\text { Visits and } \\
\text { caregivers assigned } \\
(\%)\end{array}$} & \multicolumn{2}{|c|}{$\begin{array}{c}\text { Deviation ratio } \\
(\%)\end{array}$} & \multicolumn{2}{|c|}{$\begin{array}{l}\text { Solving time } \\
(\mathrm{s})\end{array}$} & \multicolumn{2}{|c|}{$\begin{array}{c}\text { Feasible solution } \\
(\%)\end{array}$} \\
\hline & & Original & Improved & Original & Improved & Original & Improved \\
\hline MIP & - & \multicolumn{2}{|r|}{-} & \multicolumn{2}{|c|}{352.28} & \multicolumn{2}{|c|}{100} \\
\hline \multirow{11}{*}{$\begin{array}{l}\text { Matheuristic } \\
\text { Nearest Depot } \\
(N D)\end{array}$} & 0 & 0.83 & 0.17 & 1.13 & 1.17 & 92.86 & 100 \\
\hline & 10 & 0.73 & 0.31 & 0.91 & 1.14 & 89.29 & 100 \\
\hline & 20 & 1.66 & 1.12 & 0.91 & 1.23 & 91.07 & 100 \\
\hline & 30 & 3.96 & 3.90 & 0.87 & 1.05 & 94.64 & 100 \\
\hline & 40 & 6.73 & 6.49 & 0.94 & 1.17 & 87.50 & 100 \\
\hline & 50 & 7.79 & 7.37 & 0.75 & 0.74 & 89.29 & 100 \\
\hline & 60 & 8.22 & 8.30 & 0.61 & 0.66 & 91.07 & 100 \\
\hline & 70 & 13.42 & 11.96 & 0.57 & 0.68 & 78.57 & 100 \\
\hline & 80 & 15.67 & 13.07 & 0.50 & 0.64 & 78.57 & 100 \\
\hline & 90 & 18.23 & 13.18 & 0.43 & 0.64 & 76.79 & 100 \\
\hline & 100 & 22.50 & 18.38 & 0.40 & 0.53 & 60.71 & 100 \\
\hline \multirow{11}{*}{$\begin{array}{l}\text { Matheuristic } \\
\text { Isolated Nearest Depot } \\
(\text { IND) }\end{array}$} & 0 & 0.83 & 0.17 & 1.19 & 1.20 & 92.86 & 100 \\
\hline & 10 & 0.74 & 0.17 & 1.09 & 1.31 & 91.07 & 100 \\
\hline & 20 & 0.92 & 0.13 & 0.97 & 1.12 & 92.86 & 100 \\
\hline & 30 & 0.82 & 0.17 & 0.86 & 0.90 & 92.86 & 100 \\
\hline & 40 & 0.81 & 0.30 & 0.79 & 0.81 & 91.07 & 100 \\
\hline & 50 & 2.05 & 1.94 & 0.65 & 0.65 & 91.07 & 100 \\
\hline & 60 & 3.49 & 3.50 & 0.62 & 0.63 & 91.07 & 100 \\
\hline & 70 & 7.49 & 6.53 & 0.60 & 0.63 & 85.71 & 100 \\
\hline & 80 & 9.31 & 8.76 & 0.54 & 0.62 & 83.93 & 100 \\
\hline & 90 & 13.91 & 12.23 & 0.48 & 0.53 & 76.79 & 100 \\
\hline & 100 & 21.60 & 17.54 & 0.37 & 0.53 & 60.71 & 100 \\
\hline \multirow{11}{*}{$\begin{array}{l}\text { Matheuristic } \\
\text { Average Nearest Cluster } \\
(A N C)\end{array}$} & 0 & 0.83 & 0.17 & 1.14 & 1.17 & 92.86 & 100 \\
\hline & 10 & 0.71 & 0.29 & 1.05 & 1.24 & 91.07 & 100 \\
\hline & 20 & 1.34 & 0.77 & 0.92 & 1.15 & 91.07 & 100 \\
\hline & 30 & 2.00 & 1.31 & 0.85 & 0.92 & 92.86 & 100 \\
\hline & 40 & 2.16 & 1.55 & 0.79 & 0.82 & 89.29 & 100 \\
\hline & 50 & 4.24 & 4.48 & 0.63 & 0.66 & 87.50 & 100 \\
\hline & 60 & 5.97 & 5.70 & 0.61 & 0.62 & 89.29 & 100 \\
\hline & 70 & 6.75 & 6.84 & 0.57 & 0.56 & 87.50 & 100 \\
\hline & 80 & 8.01 & 7.87 & 0.53 & 0.53 & 89.29 & 100 \\
\hline & 90 & 10.43 & 9.84 & 0.45 & 0.51 & 73.21 & 100 \\
\hline & 100 & 12.52 & 12.05 & 0.37 & 0.58 & 69.64 & 100 \\
\hline \multirow{11}{*}{$\begin{array}{l}\text { Matheuristic } \\
\text { Average Nearest } \\
\text { Isolated Cluster } \\
(\text { ANIC) }\end{array}$} & 0 & 0.83 & 0.17 & 1.17 & 1.17 & 92.86 & 100 \\
\hline & 10 & 0.74 & 0.17 & 1.04 & 1.24 & 91.07 & 100 \\
\hline & 20 & 0.92 & 0.13 & 0.93 & 1.09 & 92.86 & 100 \\
\hline & 30 & 0.82 & 0.17 & 0.82 & 0.86 & 92.86 & 100 \\
\hline & 40 & 0.81 & 0.30 & 0.76 & 0.79 & 91.07 & 100 \\
\hline & 50 & 2.05 & 1.94 & 0.63 & 0.64 & 91.07 & 100 \\
\hline & 60 & 3.49 & 3.50 & 0.61 & 0.62 & 91.07 & 100 \\
\hline & 70 & 7.33 & 6.34 & 0.57 & 0.64 & 85.71 & 100 \\
\hline & 80 & 9.27 & 7.90 & 0.54 & 0.58 & 83.93 & 100 \\
\hline & 90 & 14.99 & 12.55 & 0.47 & 0.58 & 73.21 & 100 \\
\hline & 100 & 21.78 & 17.15 & 0.36 & 0.56 & 66.07 & 100 \\
\hline \multirow{4}{*}{$\begin{array}{l}\text { Matheuristic } \\
\text { Nearest Cluster Node } \\
(N C N)\end{array}$} & 0 & 0.83 & 0.17 & 1.17 & 1.16 & 92.86 & 100 \\
\hline & 10 & 0.68 & 0.17 & 1.06 & 1.23 & 94.64 & 100 \\
\hline & 20 & 1.30 & 0.77 & 0.89 & 1.06 & 89.29 & 100 \\
\hline & 30 & 1.79 & 1.19 & 0.83 & 0.98 & 91.07 & 100 \\
\hline
\end{tabular}


TABLE 4. (Continued.)

\begin{tabular}{|c|c|c|c|c|c|c|c|}
\hline \multirow[t]{2}{*}{ Solving method } & \multirow{2}{*}{$\begin{array}{l}\text { Visits and } \\
\text { caregivers assigned } \\
(\%)\end{array}$} & \multicolumn{2}{|c|}{$\begin{array}{c}\text { Deviation ratio } \\
(\%)\end{array}$} & \multicolumn{2}{|c|}{$\begin{array}{l}\text { Solving time } \\
(\mathrm{s})\end{array}$} & \multicolumn{2}{|c|}{$\begin{array}{l}\text { Feasible solution } \\
\qquad(\%)\end{array}$} \\
\hline & & Original & Improved & Original & Improved & Original & Improved \\
\hline \multirow[t]{8}{*}{ MIP } & - & \multicolumn{2}{|r|}{-} & \multicolumn{2}{|c|}{352.28} & \multicolumn{2}{|c|}{100} \\
\hline & 40 & 2.45 & 1.75 & 0.87 & 1.05 & 91.07 & 100 \\
\hline & 50 & 4.26 & 3.53 & 0.84 & 0.90 & 92.86 & 100 \\
\hline & 60 & 3.51 & 4.14 & 0.72 & 0.80 & 87.50 & 100 \\
\hline & 70 & 4.00 & 4.44 & 0.65 & 0.70 & 85.71 & 100 \\
\hline & 80 & 4.25 & 4.69 & 0.58 & 0.94 & 83.93 & 100 \\
\hline & 90 & 8.56 & 6.57 & 0.39 & 0.60 & 73.21 & 100 \\
\hline & 100 & 10.85 & 10.19 & 0.38 & 0.49 & 67.86 & 100 \\
\hline Matheuristic & 0 & 0.83 & 0.17 & 1.14 & 1.14 & 92.86 & 100 \\
\hline Nearest Isolated & 10 & 0.74 & 0.17 & 1.06 & 1.23 & 91.07 & 100 \\
\hline Cluster Node & 20 & 0.92 & 0.13 & 0.93 & 1.08 & 92.86 & 100 \\
\hline$(N I C N)$ & 30 & 0.82 & 0.17 & 0.82 & 0.86 & 92.86 & 100 \\
\hline & 40 & 0.81 & 0.30 & 0.77 & 0.79 & 91.07 & 100 \\
\hline & 50 & 2.05 & 1.94 & 0.66 & 0.65 & 91.07 & 100 \\
\hline & 60 & 3.49 & 3.50 & 0.62 & 0.63 & 91.07 & 100 \\
\hline & 70 & 7.51 & 6.52 & 0.59 & 0.68 & 85.71 & 100 \\
\hline & 80 & 9.92 & 7.76 & 0.54 & 0.83 & 83.93 & 100 \\
\hline & 90 & 15.08 & 12.73 & 0.47 & 0.54 & 75.00 & 100 \\
\hline & 100 & 21.73 & 16.68 & 0.38 & 0.69 & 62.50 & 100 \\
\hline
\end{tabular}

Concerning the reliability of the solving methods, the optimization solver finds a feasible solution for all instances while in few cases, the matheuristic-based approach cannot find one. As explained in Section 4.3, the original two-phases matheuristic from the literature may not always find a feasible solution. As a result, it is noticeable in the sub-column "Original" that the probability of obtaining a feasible solution range from around 60 to $90 \%$ depending on the proportion of visits and caregivers assigned. However, this drawback is completely removed when using the improved version of the two-phases matheuristic, where a feasible solution is always found as shown in the sub-column "Improved".

In addition, the results highlight the efficiency of the matheuristic-based approach using the improved twophases matheuristic since it provides slightly better deviation ratio with a similar computational time and a feasible solution is always found.

Regarding the medium-sized instances, similar conclusions can be drawn. In fact, the deviation ratio is overall slightly higher than for small size instances, but remains low, especially when using the "Nearest Cluster Node" assignment strategy. Furthermore, the performance of the matheuristic-based approach is near optimal when at most $60 \%$ of visits and caregivers are assigned to a home health care office. In this case, the deviation ratio remains lower than $6 \%$ on average, except for the assignment strategy "Nearest Depot" which provides the worst results.

In addition, it should be highlighted that the computational time decreases as much as the problem is made simpler by assigning visits and caregivers to the home health care offices. For instance, it takes an average of $922.14 \mathrm{~s}$ to the matheuristic-based approach using the assignment strategy "Nearest Cluster Node" to solve the 56 medium-sized instances when assigning $10 \%$ of the visits and caregivers to the home health care offices. In opposite, it takes only $119.26 \mathrm{~s}$ to the same solving method when assigning all the visits and caregivers, while the optimization solver needs $4028.96 \mathrm{~s}$. Consequently, a trade-off must be found between efficiency and fastness of the solving method.

It should also be pointed out that most of the computational time required to solve the problem is mostly spent by Gurobi on the planning of nurses and auxiliary nurses. The initial assignment steps of visits and caregivers account for only an infinitesimal portion of the computational time. 
TABLE 5. Comparison of the results of the matheuristic-based approach with the commercial optimization solver on medium-sized instances.

\begin{tabular}{|c|c|c|c|c|c|c|c|}
\hline \multirow[t]{2}{*}{ Solving method } & \multirow{2}{*}{$\begin{array}{l}\text { Visits and caregivers } \\
\text { assigned (\%) }\end{array}$} & \multicolumn{2}{|c|}{ Deviation ratio (\%) } & \multicolumn{2}{|c|}{ Solving time (s) } & \multicolumn{2}{|c|}{ Feasible solution (\%) } \\
\hline & & Original & Improved & Original & Improved & Original & Improved \\
\hline MIP & - & & - & 40 & 8.96 & & 100 \\
\hline \multirow{11}{*}{$\begin{array}{l}\text { Matheuristic } \\
\text { Nearest Depot } \\
(N D)\end{array}$} & 0 & 0.90 & 0.20 & 988.24 & 1121.16 & 89.29 & 100 \\
\hline & 10 & 1.63 & 0.86 & 924.20 & 1134.69 & 91.07 & 100 \\
\hline & 20 & 1.72 & 1.12 & 950.62 & 1099.19 & 85.71 & 100 \\
\hline & 30 & 2.25 & 1.80 & 1076.64 & 1165.77 & 85.71 & 100 \\
\hline & 40 & 5.58 & 4.80 & 1308.84 & 1426.37 & 87.50 & 100 \\
\hline & 50 & 8.97 & 8.65 & 1350.58 & 1427.00 & 91.07 & 100 \\
\hline & 60 & 10.09 & 9.44 & 1172.32 & 1259.83 & 89.29 & 100 \\
\hline & 70 & 10.54 & 10.47 & 871.47 & 954.63 & 89.29 & 100 \\
\hline & 80 & 16.13 & 15.68 & 778.76 & 895.97 & 91.07 & 100 \\
\hline & 90 & 19.67 & 19.13 & 495.55 & 654.80 & 87.50 & 100 \\
\hline & 100 & 29.52 & 27.69 & 423.71 & 493.42 & 91.07 & 100 \\
\hline \multirow{11}{*}{$\begin{array}{l}\text { Matheuristic } \\
\text { Isolated Nearest Depot } \\
(\text { IND) }\end{array}$} & 0 & 0.88 & 0.20 & 904.51 & 1120.42 & 89.29 & 100 \\
\hline & 10 & 1.13 & 0.50 & 782.42 & 1099.15 & 82.14 & 100 \\
\hline & 20 & 1.04 & 0.51 & 728.73 & 1159.15 & 83.93 & 100 \\
\hline & 30 & 1.48 & 0.87 & 1002.99 & 1166.87 & 83.93 & 100 \\
\hline & 40 & 1.68 & 1.14 & 1044.55 & 964.50 & 91.07 & 100 \\
\hline & 50 & 4.18 & 3.35 & 860.95 & 1157.67 & 85.71 & 100 \\
\hline & 60 & 4.94 & 4.45 & 888.53 & 939.10 & 89.29 & 100 \\
\hline & 70 & 11.20 & 10.59 & 722.75 & 932.32 & 87.50 & 100 \\
\hline & 80 & 13.95 & 12.92 & 672.08 & 612.04 & 89.29 & 100 \\
\hline & 90 & 20.36 & 19.66 & 596.80 & 505.85 & 91.07 & 100 \\
\hline & 100 & 28.13 & 30.30 & 372.73 & 468.14 & 91.07 & 100 \\
\hline \multirow{11}{*}{$\begin{array}{l}\text { Matheuristic } \\
\text { Aveage Nearest Cluster } \\
(A N C)\end{array}$} & 0 & 0.88 & 0.20 & 904.85 & 1120.81 & 89.29 & 100 \\
\hline & 10 & 1.78 & 0.97 & 918.48 & 1191.27 & 89.29 & 100 \\
\hline & 20 & 1.88 & 1.20 & 839.23 & 1157.99 & 87.50 & 100 \\
\hline & 30 & 1.88 & 1.42 & 1043.43 & 998.06 & 83.93 & 100 \\
\hline & 40 & 2.44 & 1.58 & 410.98 & 870.99 & 76.79 & 100 \\
\hline & 50 & 3.25 & 1.78 & 590.23 & 773.07 & 87.50 & 100 \\
\hline & 60 & 3.05 & 2.37 & 377.92 & 464.14 & 87.50 & 100 \\
\hline & 70 & 3.61 & 3.01 & 255.10 & 329.23 & 96.43 & 100 \\
\hline & 80 & 4.49 & 3.59 & 88.20 & 276.51 & 98.21 & 100 \\
\hline & 90 & 8.28 & 7.44 & 166.06 & 101.40 & 96.43 & 100 \\
\hline & 100 & 17.51 & 18.96 & 160.51 & 166.80 & 92.86 & 100 \\
\hline \multirow{11}{*}{$\begin{array}{l}\text { Matheuristic } \\
\text { Average Nearest } \\
\text { Isolated Cluster } \\
(\text { ANIC) }\end{array}$} & 0 & 0.88 & 0.20 & 906.85 & 1118.65 & 89.29 & 100 \\
\hline & 10 & 1.13 & 0.50 & 640.21 & 1086.91 & 80.36 & 100 \\
\hline & 20 & 1.02 & 0.51 & 727.52 & 1141.96 & 83.93 & 100 \\
\hline & 30 & 1.42 & 0.87 & 966.85 & 1164.23 & 83.93 & 100 \\
\hline & 40 & 1.65 & 1.14 & 672.03 & 992.82 & 87.50 & 100 \\
\hline & 50 & 3.98 & 3.35 & 640.93 & 1173.61 & 83.93 & 100 \\
\hline & 60 & 5.05 & 4.52 & 852.93 & 953.14 & 87.50 & 100 \\
\hline & 70 & 10.70 & 10.64 & 515.16 & 918.43 & 89.29 & 100 \\
\hline & 80 & 13.80 & 13.32 & 441.57 & 649.65 & 87.50 & 100 \\
\hline & 90 & 21.62 & 19.06 & 388.72 & 454.06 & 96.43 & 100 \\
\hline & 100 & 30.10 & 29.60 & 428.17 & 522.92 & 92.86 & 100 \\
\hline \multirow{5}{*}{$\begin{array}{l}\text { Matheuristic } \\
\text { Nearest Cluster Node } \\
(N C N)\end{array}$} & 0 & 0.88 & 0.20 & 904.91 & 1117.58 & 89.29 & 100 \\
\hline & 10 & 0.98 & 0.44 & 922.14 & 1235.21 & 85.71 & 100 \\
\hline & 20 & 1.13 & 0.60 & 955.69 & 1137.97 & 85.71 & 100 \\
\hline & 30 & 1.27 & 0.74 & 978.18 & 1068.29 & 83.93 & 100 \\
\hline & 40 & 1.32 & 0.68 & 861.42 & 978.15 & 82.14 & 100 \\
\hline
\end{tabular}


TABLE 5. (Continued.)

\begin{tabular}{|c|c|c|c|c|c|c|c|}
\hline \multirow[t]{2}{*}{ Solving method } & \multirow{2}{*}{$\begin{array}{l}\text { Visits and caregivers } \\
\text { assigned (\%) }\end{array}$} & \multicolumn{2}{|c|}{ Deviation ratio (\%) } & \multicolumn{2}{|c|}{ Solving time (s) } & \multicolumn{2}{|c|}{ Feasible solution (\%) } \\
\hline & & Original & Improved & Original & Improved & Original & Improved \\
\hline \multirow[t]{7}{*}{ MIP } & - & & - & \multicolumn{2}{|c|}{4028.96} & \multicolumn{2}{|c|}{100} \\
\hline & 50 & 1.57 & 0.83 & 616.80 & 915.43 & 80.36 & 100 \\
\hline & 60 & 1.89 & 1.56 & 646.36 & 937.04 & 89.29 & 100 \\
\hline & 70 & 3.23 & 2.35 & 276.52 & 525.62 & 87.50 & 100 \\
\hline & 80 & 5.49 & 3.55 & 303.62 & 366.56 & 98.21 & 100 \\
\hline & 90 & 9.68 & 8.32 & 57.62 & 128.51 & 98.21 & 100 \\
\hline & 100 & 14.13 & 14.74 & 119.26 & 143.89 & 94.64 & 100 \\
\hline Matheuristic & 0 & 0.88 & 0.20 & 906.99 & 1117.94 & 89.29 & 100 \\
\hline Nearest Isolated & 10 & 1.13 & 0.50 & 649.19 & 1088.24 & 80.36 & 100 \\
\hline Cluster Node & 20 & 1.02 & 0.51 & 727.60 & 1146.66 & 83.93 & 100 \\
\hline \multirow[t]{8}{*}{$(N I C N)$} & 30 & 1.44 & 0.87 & 972.93 & 1164.07 & 83.93 & 100 \\
\hline & 40 & 1.62 & 1.14 & 980.08 & 962.42 & 92.86 & 100 \\
\hline & 50 & 3.96 & 3.35 & 929.65 & 1152.35 & 85.71 & 100 \\
\hline & 60 & 5.09 & 4.65 & 531.88 & 1066.41 & 85.71 & 100 \\
\hline & 70 & 10.90 & 10.67 & 632.74 & 894.02 & 91.07 & 100 \\
\hline & 80 & 13.42 & 12.79 & 300.55 & 657.88 & 87.50 & 100 \\
\hline & 90 & 19.74 & 20.10 & 292.02 & 525.28 & 89.29 & 100 \\
\hline & 100 & 29.60 & 27.68 & 374.74 & 507.76 & 94.64 & 100 \\
\hline
\end{tabular}

Finally, while the optimization solver provides good results with a high reliability, it has a very high computation time. In opposite, the matheuristic-based approach provides good results and reliability with a very low computation time.

Secondly, the lower bounds obtained by Lagrangian relaxation (LR) must be compared with the best-found solution for each instance and with the lower bound obtained by Gurobi, in order to assess the quality of the lower bound obtained by Lagrangian relaxation. Computational results are presented in Table 6 .

The column "Instance name" indicates the instance used for the experiment. Instance name ending with the suffix "_25" indicates that the considered instance is a small size instance. In opposite, instances name ending with the suffix "_50" indicates that they are medium-sized instances.

The column "Best-found solution" represents the best solution obtained among all the experiments performed by the matheuristic-based approach and by the optimization solver. A star mark $\left(^{*}\right)$ is used in this column to indicate that the solution has been proven to be optimal by the solver.

Moreover, the columns "Lower bound MIP" and "Lower bound LR" respectively indicate the lower bounds obtained for each instance by the commercial solver Gurobi and by the Lagrangian relaxation. As a result, the two columns "Gap (\%)" indicate the deviation ratio between the best-found solution and the lower bound obtained either by the commercial solver or by the Lagrangian relaxation. The lower the deviation ratio is and the better the result is.

For each instance, the best result is highlighted in gray to distinguish which method has provided the best lower bound.

Based on the results provided by the tables, the Lagrangian relaxation provides good results compared to the optimization solver.

Indeed, with the same time limit (i.e. two hours), the lower bound obtained by the Lagrangian relaxation is overall higher than the one obtained by the commercial solver Gurobi.

Regarding the small size instances, the performances of both algorithms are quite similar. For instance, the lower bound obtained by the Lagrangian relaxation is much better for the instance RC108_25 (the deviation ratio with the best-found solution is only $0.65 \%$ for the LR while it is equal to $4.20 \%$ for the commercial solver). 
TABLE 6. Comparison of the lower bound obtained by the Lagrangian relaxation with the best-found solution and the lower bound obtained by Gurobi.

\begin{tabular}{|c|c|c|c|c|c|c|c|c|c|c|c|}
\hline $\begin{array}{l}\text { Instance } \\
\text { name }\end{array}$ & $\begin{array}{l}\text { Best } \\
\text { found } \\
\text { solution }\end{array}$ & $\begin{array}{l}\text { Lower } \\
\text { bound } \\
\text { MIP }\end{array}$ & $\begin{array}{l}\text { Gap } \\
(\%)\end{array}$ & $\begin{array}{l}\text { Lower } \\
\text { bound } \\
\text { LR }\end{array}$ & $\begin{array}{l}\text { Gap } \\
(\%)\end{array}$ & $\begin{array}{l}\text { Instance } \\
\text { name }\end{array}$ & $\begin{array}{l}\text { Best } \\
\text { found } \\
\text { solution }\end{array}$ & $\begin{array}{l}\text { Lower } \\
\text { bound } \\
\text { MIP }\end{array}$ & $\begin{array}{l}\text { Gap } \\
(\%)\end{array}$ & $\begin{array}{l}\text { Lower } \\
\text { bound } \\
\text { LR }\end{array}$ & $\begin{array}{l}\text { Gap } \\
(\%)\end{array}$ \\
\hline C101_25 & $267.16^{*}$ & 267.16 & 0 & 267.16 & 0 & C101_50 & $535.59^{*}$ & 535.59 & 0 & 535.59 & 0 \\
\hline C102_25 & $242.80^{*}$ & 242.80 & 0 & 242.80 & 0 & C102_50 & $524.63^{*}$ & 524.62 & 0 & 524.63 & 0 \\
\hline C103_25 & $224.76^{*}$ & 224.76 & 0 & 224.76 & 0 & C103_50 & 512.01 & 460.37 & 11.22 & 465.12 & 10.08 \\
\hline C104_25 & $219.19^{*}$ & 219.19 & 0 & 219.19 & 0 & C104_50 & 492.69 & 418.70 & 17.67 & 471.90 & 4.41 \\
\hline C105_25 & $263.76^{*}$ & 263.76 & 0 & 263.76 & 0 & C105_50 & $535.59^{*}$ & 535.59 & 0 & 535.59 & 0 \\
\hline C106_25 & $267.16^{*}$ & 267.16 & 0 & 267.16 & 0 & C106_50 & $535.59 *$ & 535.59 & 0 & 535.59 & 0 \\
\hline C107_25 & $256.06^{*}$ & 256.06 & 0 & 256.06 & 0 & C107_50 & $535.59 *$ & 535.59 & 0 & 535.59 & 0 \\
\hline C108_25 & $255.52^{*}$ & 255.52 & 0 & 255.52 & 0 & C108_50 & $521.20^{*}$ & 521.20 & 0 & 521.20 & 0 \\
\hline C109_25 & $219.59^{*}$ & 219.59 & 0 & 219.59 & 0 & C109_50 & 514.06 & 503.54 & 2.09 & 502.01 & 2.40 \\
\hline C201_25 & $341.28^{*}$ & 341.28 & 0 & 341.28 & 0 & C201_50 & $595.88^{*}$ & 595.88 & 0 & 595.88 & 0 \\
\hline C202_25 & $315.88^{*}$ & 315.88 & 0 & 315.88 & 0 & C202_50 & $564.81^{*}$ & 564.76 & 0.01 & 564.81 & 0 \\
\hline C203_25 & $315.88^{*}$ & 315.88 & 0 & 315.88 & 0 & C203_50 & $551.27^{*}$ & 551.22 & 0.01 & 548.79 & 0.45 \\
\hline C204_25 & $304.03^{*}$ & 304.03 & 0 & 304.03 & 0 & C204_50 & 551.86 & 507.46 & 8.75 & 540.55 & 2.09 \\
\hline C205_25 & $339.48^{*}$ & 339.48 & 0 & 339.48 & 0 & C205_50 & 584.04* & 584.04 & 0 & 584.04 & 0 \\
\hline C206_25 & $339.48^{*}$ & 339.48 & 0 & 339.48 & 0 & C206_50 & $580.64^{*}$ & 580.61 & 0.01 & 580.64 & 0 \\
\hline C207_25 & $338.79^{*}$ & 338.79 & 0 & 338.79 & 0 & C207_50 & 572.37 & 562.45 & 1.76 & 568.68 & 0.65 \\
\hline C208_25 & $335.10^{*}$ & 335.10 & 0 & 335.10 & 0 & C208_50 & $574.29 *$ & 574.24 & 0.01 & 574.29 & 0 \\
\hline R101_25 & $718.53^{*}$ & 718.53 & 0 & 718.53 & 0 & R101_50 & $1137.53^{*}$ & 1137.53 & 0 & 1137.53 & 0 \\
\hline R102_25 & $599.41^{*}$ & 599.41 & 0 & 599.41 & 0 & R102_50 & 940.86* & 940.79 & 0.01 & 940.86 & 0 \\
\hline R103_25 & $541.71^{*}$ & 541.71 & 0 & 541.71 & 0 & R103_50 & 877.85 & 865.49 & 1.43 & 859.95 & 2.08 \\
\hline R104_25 & $528.42^{*}$ & 528.42 & 0 & 528.42 & 0 & R104_50 & 793.86 & 771.56 & 2.89 & 787.47 & 0.81 \\
\hline R105_25 & $585.30^{*}$ & 585.30 & 0 & 585.30 & 0 & R105_50 & $957.50 *$ & 957.50 & 0 & 957.50 & 0 \\
\hline R106_25 & $545.34^{*}$ & 545.34 & 0 & 545.34 & 0 & R106_50 & 871.13 & 858.46 & 1.47 & 857.44 & 1.60 \\
\hline R107_25 & $521.34^{*}$ & 521.34 & 0 & 521.34 & 0 & R107_50 & 829.16 & 816.01 & 1.61 & 816.14 & 1.60 \\
\hline R108_25 & $513.32^{*}$ & 513.32 & 0 & 513.32 & 0 & R108_50 & 793.86 & 758.61 & 4.65 & 748.97 & 5.99 \\
\hline R109_25 & $548.64^{*}$ & 548.64 & 0 & 548.64 & 0 & R109_50 & $842.33^{*}$ & 842.25 & 0.01 & 838.70 & 0.43 \\
\hline R110_25 & $535.75^{*}$ & 535.75 & 0 & 535.75 & 0 & R110_50 & 833.10 & 798.62 & 4.32 & 795.11 & 4.78 \\
\hline R111_25 & $521.34^{*}$ & 521.34 & 0 & 521.34 & 0 & R111_50 & 824.09 & 800.36 & 2.97 & 812.99 & 1.37 \\
\hline R112_25 & $504.11^{*}$ & 504.11 & 0 & 504.11 & 0 & R112_50 & 788.35 & 750.85 & 4.99 & 740.76 & 6.42 \\
\hline R201_25 & $529.56^{*}$ & 529.56 & 0 & 529.56 & 0 & R201_50 & $855.36^{*}$ & 855.36 & 0 & 855.36 & 0 \\
\hline R202_25 & $498.57^{*}$ & 498.57 & 0 & 498.57 & 0 & R202_50 & 793.75* & 793.73 & 0 & 793.75 & 0 \\
\hline R203_25 & $486.32^{*}$ & 486.32 & 0 & 486.32 & 0 & R203_50 & 758.85 & 739.58 & 2.60 & 755.85 & 0.40 \\
\hline R204_25 & $474.90^{*}$ & 474.90 & 0 & 474.90 & 0 & R204_50 & 714.91 & 699.96 & 2.13 & 710.98 & 0.55 \\
\hline R205_25 & $489.52^{*}$ & 489.52 & 0 & 489.52 & 0 & R205_50 & 785.97 & 780.12 & 0.75 & 785.97 & 0 \\
\hline R206_25 & $474.90^{*}$ & 474.90 & 0 & 474.90 & 0 & R206_50 & $758.95^{*}$ & 758.88 & 0.01 & 758.95 & 0 \\
\hline R207_25 & $474.90^{*}$ & 474.90 & 0 & 474.90 & 0 & R207_50 & 739.49 & 730.02 & 1.30 & 736.33 & 0.43 \\
\hline R208_25 & $474.90^{*}$ & 474.90 & 0 & 474.90 & 0 & R208_50 & 704.10 & 692.07 & 1.74 & 701.33 & 0.40 \\
\hline R209_25 & $489.52^{*}$ & 489.52 & 0 & 489.52 & 0 & R209_50 & 743.32 & 732.52 & 1.47 & 691.96 & 7.42 \\
\hline R210_25 & $486.32^{*}$ & 486.32 & 0 & 486.32 & 0 & R210_50 & $761.22 *$ & 761.22 & 0 & 761.22 & 0 \\
\hline R211_25 & $474.90^{*}$ & 474.90 & 0 & 474.90 & 0 & R211_50 & 724.97 & 692.72 & 4.66 & 690.44 & 5.00 \\
\hline RC101_25 & $468.90^{*}$ & 468.90 & 0 & 468.90 & 0 & RC101_50 & $908.19^{*}$ & 908.11 & 0.01 & 908.19 & 0 \\
\hline RC102_25 & $449.26^{*}$ & 449.26 & 0 & 449.26 & 0 & RC102_50 & 882.31* & 882.31 & 0 & 882.31 & 0 \\
\hline RC103_25 & $430.96^{*}$ & 430.96 & 0 & 430.96 & 0 & RC103_50 & 867.73 & 734.23 & 18.18 & 816.78 & 6.24 \\
\hline RC104_25 & $425.19 *$ & 425.19 & 0 & 425.19 & 0 & RC104_50 & 799.52 & 660.63 & 21.02 & 794.80 & 0.59 \\
\hline RC105_25 & $510.08^{*}$ & 510.08 & 0 & 510.08 & 0 & RC105_50 & $912.04^{*}$ & 912.04 & 0 & 912.04 & 0 \\
\hline RC106_25 & $449.65^{*}$ & 449.65 & 0 & 449.65 & 0 & RC106_50 & $816.66^{*}$ & 816.58 & 0.01 & 813.47 & 0.39 \\
\hline RC107_25 & $464.82^{*}$ & 464.78 & 0.01 & 464.82 & 0 & RC107_50 & 837.52 & 696.15 & 20.31 & 828.99 & 1.03 \\
\hline RC108_25 & 464.82 & 446.07 & 4.20 & 461.82 & 0.65 & RC108_50 & 810.85 & 683.12 & 18.70 & 721.05 & 12.45 \\
\hline RC201_25 & $466.59^{*}$ & 466.59 & 0 & 466.59 & 0 & RC201_50 & $845.82 *$ & 845.82 & 0 & 845.82 & 0 \\
\hline RC202_25 & $449.08^{*}$ & 449.08 & 0 & 449.08 & 0 & RC202_50 & $835.64 *$ & 835.64 & 0 & 835.64 & 0 \\
\hline RC203_25 & $463.68^{*}$ & 463.68 & 0 & 463.68 & 0 & RC203_50 & 828.92 & 725.84 & 14.20 & 804.38 & 3.05 \\
\hline RC204_25 & $456.34^{*}$ & 456.29 & 0.01 & 456.34 & 0 & RC204_50 & 841.44 & 719.75 & 16.91 & 832.37 & 1.09 \\
\hline RC205_25 & $450.28^{*}$ & 450.28 & 0 & 450.28 & 0 & RC205_50 & $837.37^{*}$ & 837.37 & 0 & 837.37 & 0 \\
\hline RC206_25 & $446.70^{*}$ & 446.70 & 0 & 446.70 & 0 & RC206_50 & $821.53^{*}$ & 821.45 & 0.01 & 814.59 & 0.85 \\
\hline RC207_25 & $456.03^{*}$ & 456.03 & 0 & 456.03 & 0 & RC207_50 & 845.27 & 735.25 & 14.96 & 829.74 & 1.87 \\
\hline RC208_25 & 443.12 & 443.08 & 0.01 & 439.60 & 0.80 & RC208_50 & 884.19 & 741.27 & 19.28 & 866.91 & 1.99 \\
\hline
\end{tabular}


TABLE 7. Comparison of the results of the matheuristic-based approach with the lower bound obtained by Lagrangian relaxation on medium-sized instances.

\begin{tabular}{|c|c|c|c|}
\hline \multirow[t]{2}{*}{ Solving method } & \multirow{2}{*}{$\begin{array}{l}\text { Visits and caregivers } \\
\text { assigned }(\%)\end{array}$} & \multicolumn{2}{|c|}{ Error ratio (\%) } \\
\hline & & Original & Improved \\
\hline \multirow{11}{*}{$\begin{array}{l}\text { Matheuristic } \\
\text { Nearest Depot } \\
(N D)\end{array}$} & 0 & 2.47 & 1.95 \\
\hline & 10 & 2.97 & 2.61 \\
\hline & 20 & 3.14 & 2.87 \\
\hline & 30 & 4.08 & 3.56 \\
\hline & 40 & 7.34 & 6.60 \\
\hline & 50 & 10.96 & 10.54 \\
\hline & 60 & 12.01 & 11.33 \\
\hline & 70 & 12.51 & 12.38 \\
\hline & 80 & 18.15 & 17.69 \\
\hline & 90 & 21.28 & 21.17 \\
\hline & 100 & 31.74 & 29.85 \\
\hline \multirow{11}{*}{$\begin{array}{l}\text { Matheuristic } \\
\text { Isolated Nearest Depot } \\
(\text { IND) }\end{array}$} & 0 & 2.46 & 1.95 \\
\hline & 10 & 2.51 & 2.25 \\
\hline & 20 & 2.34 & 2.26 \\
\hline & 30 & 2.95 & 2.62 \\
\hline & 40 & 3.45 & 2.90 \\
\hline & 50 & 5.57 & 5.14 \\
\hline & 60 & 6.75 & 6.26 \\
\hline & 70 & 12.87 & 12.52 \\
\hline & 80 & 16.03 & 14.88 \\
\hline & 90 & 22.52 & 21.69 \\
\hline & 100 & 30.40 & 32.49 \\
\hline \multirow{11}{*}{$\begin{array}{l}\text { Matheuristic } \\
\text { Average Nearest Cluster } \\
(A N C)\end{array}$} & 0 & 2.46 & 1.95 \\
\hline & 10 & 3.38 & 2.72 \\
\hline & 20 & 3.45 & 2.95 \\
\hline & 30 & 3.67 & 3.18 \\
\hline & 40 & 3.72 & 3.34 \\
\hline & 50 & 4.71 & 3.54 \\
\hline & 60 & 4.71 & 4.14 \\
\hline & 70 & 5.48 & 4.78 \\
\hline & 80 & 6.32 & 5.37 \\
\hline & 90 & 10.16 & 9.29 \\
\hline & 100 & 19.26 & 21.05 \\
\hline \multirow{11}{*}{$\begin{array}{l}\text { Matheuristic } \\
\text { Average Nearest } \\
\text { Isolated Cluster } \\
(A N I C)\end{array}$} & 0 & 2.46 & 1.95 \\
\hline & 10 & 2.50 & 2.25 \\
\hline & 20 & 2.32 & 2.26 \\
\hline & 30 & 2.89 & 2.62 \\
\hline & 40 & 2.99 & 2.90 \\
\hline & 50 & 5.34 & 5.14 \\
\hline & 60 & 6.77 & 6.34 \\
\hline & 70 & 12.65 & 12.60 \\
\hline & 80 & 15.87 & 15.27 \\
\hline & 90 & 23.71 & 21.09 \\
\hline & 100 & 32.12 & 31.86 \\
\hline \multirow{7}{*}{$\begin{array}{l}\text { Matheuristic } \\
\text { Nearest Cluster Node } \\
(N C N)\end{array}$} & 0 & 2.46 & 1.95 \\
\hline & 10 & 2.45 & 2.19 \\
\hline & 20 & 2.87 & 2.36 \\
\hline & 30 & 3.10 & 2.50 \\
\hline & 40 & 2.85 & 2.43 \\
\hline & 50 & 2.98 & 2.59 \\
\hline & 60 & 3.39 & 3.33 \\
\hline
\end{tabular}


TABLE 7. (Continued.)

\begin{tabular}{lrrr}
\hline \hline \multirow{2}{*}{ Solving method } & \multirow{2}{*}{$\begin{array}{l}\text { Visits and caregivers } \\
\text { assigned (\%) }\end{array}$} & \multicolumn{2}{c}{ Error ratio (\%) } \\
\cline { 3 - 4 } & & Original & Improved \\
\hline & 70 & 4.67 & 4.14 \\
& 80 & 7.36 & 5.35 \\
& 90 & 11.44 & 10.22 \\
\hline Matheuristic & 100 & 16.30 & 16.67 \\
Nearest Isolated & 0 & 2.46 & 1.95 \\
Cluster Node & 20 & 2.50 & 2.25 \\
(NICN) & 30 & 2.32 & 2.26 \\
& 40 & 2.90 & 2.62 \\
& 50 & 3.37 & 2.90 \\
& 60 & 5.65 & 5.14 \\
& 70 & 6.58 & 6.46 \\
& 80 & 12.87 & 12.61 \\
& 90 & 15.41 & 14.75 \\
& 100 & 21.48 & 22.14 \\
& & 31.44 & 29.83 \\
\hline
\end{tabular}

In opposite, the lower bound obtained by Gurobi is better than the one obtained by Lagrangian relaxation for the instance RC208_25. Concerning all other small size instances, both methods provide similar results.

As for the middle-sized instances, the gap between the two algorithms is more visible. In this case, the efficiency of the Lagrangian relaxation is noticeable with respect to the commercial solver. In fact, the lower bound obtained by the Lagrangian relaxation is better in 33 out of 64 instances while the commercial solver performs better in only 15 middle-sized instances.

Overall, the lower bound obtained by the Lagrangian relaxation is better than the one obtained by the commercial solver in 33 out of 112 instances. In opposite, the commercial solver performs better in 15 out of 112 instances. Finally, both methods obtain the same lower bound in 64 out of 112 instances.

As a result, the computational results highlight the efficiency of the proposed Lagrangian relaxation. Overall, it maintains a low deviation ration from the best solution found on the test instances. Lower bounds obtained by Lagrangian relaxation may be useful to better apprehend the effectiveness of the matheuristic-based approach.

Lastly, the results of the matheuristic-based approach are compared with the lower bound obtained by Lagrangian relaxation on middle-sized instances, where Gurobi can find the optimal solution for only $50 \%$ of the instances. The aim is to better assess the efficiency of the matheuristic-based approach by comparing the results obtained with a lower bound. Computational results are presented in Table 7.

The column "Error ratio (\%)" represents the gap between the matheuristic-based approach compared to the lower bound obtained by Lagrangian relaxation (LR). The lower the error ratio is and the better the result is. Consequently, the error ratio is computed as follows:

$$
\text { Error ratio }=\frac{\text { Matheuristic }- \text { LR }}{\text { LR }}
$$

The efficiency of the matheuristic-based approach is noticeable by observing the computational results. When considering the use of the "Nearest Cluster Node" assignment strategy which was providing the best results when compared to the optimization solver, the matheuristic-based approach maintains a low error ratio with the lower bound. Indeed, the average error ratios of the matheuristic-based approach are no more than 16\%, which relates that the performance of the suggested approach is relevant. Furthermore, the error ratio can be maintained at a low-level (maximum $5-7 \%$ ) when assigning at most $80 \%$ of the visits and caregivers to the home health care offices.

In addition, the matheuristic-based approach has been evaluated on instances of up to 50 patients. Since more than $62 \%$ of the home health care structures support less than 50 patients (1313 out of 2095 structures in France 
in 2008 according to Bertrand [4], the matheuristic-based approach could then be applied to an important part of home health care offices in the French territory.

Finally, the latter results aim at verifying the efficiency of the suggested matheuristic-based approach. The matheuristic-based approach maintains a low deviation ratio either with the optimal solutions or the lower bounds obtained by Lagrangian relaxation. In addition, the assignment strategy "Nearest Cluster Node" provides the best results. Furthermore, the improvements applied to the original two-phase matheuristic from the literature have made it possible to obtain a feasible solution in all cases, which was a major drawback of the original resolution method.

Consequently, the suggested matheuristic-based approach provides a great balance between efficiency, fastness, and reliability.

\section{Conclusion}

In this paper, a model for the multi-depot home health care assignment, routing, and scheduling problem without prior assignment of caregivers to the home health care offices is presented. The problem is modeled in order to minimize the traveling time of the caregivers. By avoiding upstream assignment of visits and caregivers to offices, the model aims at better optimizing the home health care problem and obtaining lower caregivers' traveling time by an improved assignment of caregivers to offices. Furthermore, this modeling is useful when the geographical distribution of patients to be treated is growing and the home health care structures must be spread across several offices.

In addition, a matheuristic-based approach is proposed to solve the model. First, the assignment of the visits to the home health care offices is performed, followed by the assignment of caregivers to the home health care offices. Various assignment strategies are suggested for assigning visits to the home health care offices. Subsequently, the caregivers are assigned to the offices, in proportion to the number of visits assigned to each office. Secondly, the routing and scheduling phase is performed using a two-phases matheuristic from the literature, which computes the planning of the nurses first and the auxiliary nurses next. While the original two-phases matheuristic may not always find feasible planning, an improvement procedure is also presented to overcome this disadvantage. Finally, a lower bound obtained by Lagrangian relaxation is also presented.

Computational results highlight the efficiency of the suggested matheuristic-based approach which provides great results, especially when using the assignment strategy named "Nearest Cluster Node". Indeed, this assignment strategy maintains the lowest deviation ratio with the results obtained by the commercial solver. The deviation ration remains lower than around $4 \%$ when at most $80 \%$ of visits and caregivers are assigned to the home health care offices. In addition, the matheuristic-based approach is much faster than the commercial solver since it can reduce between 4 and 40 times the computational time needed compared to the optimization solver. Consequently, the suggested matheuristic-based approach provides a great balance between efficiency, fastness, and reliability.

In future works, we plan to improve the suggested matheuristic-based approach with other assignment strategies for assigning visits to the home health care offices. In particular, we expect to take into account not only the traveling time but also the time window of caregivers and patients in the computation of the assignment cost.

Acknowledgements. This work is supported by the Franche-Comté region in France (research project OptimHaD) under grant 2015C-4949.

\section{REFERENCES}

[1] J.F. Bard and H.W. Purnomo, Cyclic preference scheduling of nurses using a Lagrangian-based heuristic. J. Scheduling 10 (2007) 5-23.

[2] J.F. Bard, Y. Shao and H. Wang, Weekly scheduling models for traveling therapists. Socio Econ. Planning Sci. 47 (2013) 191-204. 
[3] S.V. Begur, D.M. Miller and J.R. Weaver, An integrated spatial DSS for scheduling and routing home-health-care nurses. Interfaces 27 (1997) 35-48.

[4] D. Bertrand, Les services de soins infirmiers à domicile en 2008. Études et résultats 739 (2010).

[5] K. Braekers, R.F. Hartl, S.N. Parragh and F. Tricoire, A bi-objective home care scheduling problem: analyzing the trade-off between costs and client inconvenience. Eur. J. Oper. Res. 248 (2016) 428-443.

[6] C. Chen, Z. Rubinstein, S. Smith and H.C. Lau, Tackling large-scale home health care delivery problem with uncertainty. In: International Conference on Automated Planning and Scheduling (ICAPS-17). Pittsburgh, PE (2017).

[7] E. Cheng and J.L. Rich, A home health care routing and scheduling problem. Technical report CAAM TR98-04, Rice University, Houston, TX (1998). http://citeseerx.ist.psu.edu/viewdoc/summary.

[8] M. Cissé, S. Yalçındağ, Y. Kergosien, E. Şahin, C. Lenté and A. Matta, Or problems related to home health care: a review of relevant routing and scheduling problems. Oper. Res. Health Care 13-14 (2017) 1-22.

[9] J. Decerle, O. Grunder, A.H.E. Hassani and O. Barakat, A two-phases matheuristic for the home care routing and scheduling problem. 8th IFAC Conference on Manufacturing Modelling, Management and Control MIM 2016. IFAC-PapersOnLine 49 (2016) 1484-1489.

[10] J. Decerle, O. Grunder, A.H. El Hassani and O. Barakat, A memetic algorithm for a home health care routing and scheduling problem. Oper. Res. Health Care 16 (2018) 59-71.

[11] J. Desrosiers, M. Sauvé and F. Soumis, Lagrangian relaxation methods for solving the minimum fleet size multiple traveling salesman problem with time windows. Manage. Sci. 34 (1988) 1005-1022.

[12] M. Di Mascolo, M.-L. Espinouse and C.E. Ozkan, Synchronization between human resources in home health care context, edited by A. Matta, J. Li, E. Sahin, E. Lanzarone and J. Fowler. In: Proceedings of the International Conference on Health Care Systems Engineering. Springer, New York, NY (2014) 73-86.

[13] G. Du, L. Zheng and X. Ouyang, Real-time scheduling optimization considering the unexpected events in home health care. J. Comb. Optim. 37 (2019) 196-220.

[14] P.M. Duque, M. Castro, K. Sörensen and P. Goos, Home care service planning. The case of Landelijke Thuiszorg. Eur. J. Oper. Res. 243 (2015) 292-301.

[15] A. Errarhout, S. Kharraja and I. Zorkani, Caregivers' assignment problem in home health care structures. In: Proceedings of 2013 International Conference on Industrial Engineering and Systems Management (IESM) (2013) 1-8.

[16] P. Eveborn, P. Flisberg and M. Rönnqvist, Laps care - an operational system for staff planning of home care. Eur. J. Oper. Res. 171 (2006) 962-976.

[17] C. Fikar and P. Hirsch, Home health care routing and scheduling: a review. Comput. Oper. Res. 77 (2017) $86-95$.

[18] M.L. Fisher, The Lagrangian relaxation method for solving integer programming problems. Manage. Sci. 27 (1981) 1-18.

[19] I. Gurobi Optimization, Gurobi optimizer reference manual (2016). http://www.gurobi.com.

[20] M. Held, P. Wolfe and H.P. Crowder, Validation of subgradient optimization. Math. Program. 6 (1974) 62-88.

[21] E. Lanzarone and A. Matta, Robust nurse-to-patient assignment in home care services to minimize overtimes under continuity of care. Oper. Res. Health Care 3 (2014) 48-58.

[22] R. Liu, Y. Tao and X. Xie, An adaptive large neighborhood search heuristic for the vehicle routing problem with time windows and synchronized visits. Comput. Oper. Res. 101 (2019) 250-262.

[23] D.S. Mankowska, F. Meisel and C. Bierwirth, The home health care routing and scheduling problem with interdependent services. Health Care Manage. Sci. 17 (2014) 15-30.

[24] S. Nickel, M. Schröder and J. Steeg, Mid-term and short-term planning support for home health care services. Eur. J. Oper. Res. 219 (2012) 574-587.

[25] S.N. Parragh and K.F. Doerner, Solving routing problems with pairwise synchronization constraints. Cent. Eur. J. Oper. Res. 26 (2018) 443-464.

[26] M.S. Rasmussen, T. Justesen, A. Dohn and J. Larsen, The home care crew scheduling problem: preference-based visit clustering and temporal dependencies. Eur. J. Oper. Res. 219 (2012) 598-610.

[27] C. Rodriguez, T. Garaix, X. Xie and V. Augusto, Staff dimensioning in homecare services with uncertain demands. Int. J. Prod. Res. 53 (2015) 7396-7410.

[28] Y. Shi, T. Boudouh and O. Grunder, A robust optimization for a home health care routing and scheduling problem with consideration of uncertain travel and service times. Transp. Res. E: Logist. Transp. Rev. 128 (2019) 52-95.

[29] M.M. Solomon, Algorithms for the vehicle routing and scheduling problems with time window constraints. Oper. Res. 35 (1987) 254-265.

[30] P. Toth and D. Vigo, The Vehicle Routing Problem. Society for Industrial and Applied Mathematics, Philadelphia, PA (2001) 363.

[31] A. Trautsamwieser, M. Gronalt and P. Hirsch, Securing home health care in times of natural disasters. OR Spect. 33 (2011) $787-813$.

[32] M. Villeneuve and J. MacDonald, Toward 2020: visions for nursing setting the stage for the future. Can. Nurse 102 (2006) 22.

[33] S. Yalçındağ, A. Matta, E. Şahin and J.G. Shanthikumar, The patient assignment problem in home health care: using a data-driven method to estimate the travel times of care givers. Flexible Serv. Manuf. J. 28 (2016) 304-335. 